\title{
The Relation between The Red Cell Distribution Width and Coronary Artery Calcium Scoring in Diabetic Patients Undergoing Coronary CT Angiography
}

\author{
Mostafa Mohamad Assy ", Marwa Fathy Khalil, Ahmed M. Alaa eldin \\ Radiodiagnosis Department, Faculty of Medicine, Zagazig University, Zagazig, Egypt
}

\author{
*Corresponding author \\ Mostafa Mohamad Assy, \\ Department of \\ Radiodiagnosis, Zagazig \\ University, Zagazig, Egypt
}

Email:

Mostafa83assy@gmail.com

$\begin{array}{ll}\text { Submit Date } & 2019-08-25 \\ \text { Revise Date } & 2019-11-02 \\ \text { Accept Date } & 2019-11-05\end{array}$

\section{ABSTRACT}

Background: This study was done to assess the relation between red cell distribution width (RDW) and coronary artery calcium score (CACS) in the diabetic patients undergoing coronary $\mathrm{CT}$ angiography and to assess the relation between RDW and severity of coronary artery disease. The objectives are to identify the role of red cell distribution width (RDW) in predicting coronary artery disease complexity and to evaluate the relationship between RDW and calcium score in diabetic patients. Methods: Thirty diabetic patients were subjected to; history taking, complete physical examination, laboratory investigations including RDW (SD \& CV) as a routine examination in complete blood count, erythrocyte sedimentation rate (ESR), lipid profile. Then multidetector CT was done for coronary artery examination and calcium scoring. Results: higher RDW (SD and CV) were associated with higher calcium score and the presence of greater coronary complexity of coronary artery disease. Cut off value of RDW to presence of coronary artery disease was $11.85 \%$ and RDW (SD) cut off was $39.5 \mathrm{fl}$, while cut off value of RDW (CV) to predict high calcium score (more than 100 ) was $12,95 \%$ and RDW (SD) more than 40.5 fl.Conclusions: a greater baseline RDW (CV and SD) value may be used as an indicator of CAD and high calcium score.

Keywords: Coronary artery disease (CAD); Multidetector CT (MDCT); red cell distribution width (RDW); coefficient variation (CV) coronary artery calcium scoring (CACS).

\section{INTRODUCTION}

Coronary artery disease (CAD) is an
essential cause of death in western countries and it is becoming a main cause of death in developing countries. This increase may be due to the rising prevalence of many CAD risk factors, such as diabetes, which is one of the most important risk factors [1].

Red blood cell distribution width (RDW) is a parameter routinely measured as a part of complete blood count (CBC), its use limited to the differential diagnosis of anemia [2]. Early diagnosis of CAD is usually considered as a major healthcare priority. Coronary artery calcium scoring (CACS) using coronary computed tomography angiography (CCTA) has been used to provide an early diagnosis of CAD [3].

Numerous studies in recent years have observed the importance of RDW as a predictor of poor clinical outcomes in several diseases, including CAD [3- 6]. In this study, we investigated the relationship between RDW and coronary artery calcium score (CACS) in diabetic patients.

\section{METHODS}

\section{Ethical statement}

This prospective study was conducted according to international guidelines that approved by the Research Ethics Committee. Informed consents were obtained from all patients prior to the study. We followed the ethical principles of the Declaration of Helsinki during the preparation of this study.

Study population

This cross-sectional study was carried out between January 2018 and March 2019. We initially collected 30 consecutive diabetic patients with chest pain. Inclusion criteria were (a) diabetic patient of both sexes, and (b) chest pain with or without history of CAD. Exclusion criteria were (a) hematological 
disorder: RDW correlates negatively with hemoglobin level, (b) thyroid disease: hyperthyroidism is associated with increased RDW, while hypothyroidism does the reverse, (c) pregnancy, (d) patients not suitable for MDCT or not suitable for contrast like those with allergy to contrast and renal failure, (e) poor general condition, (f) patient refusal, (g) previous percutaneous coronary intervention (PCI) or coronary artery bypass graft (CABG), (h) evidence of heart failure, and (i) systemic inflammatory disease. The final cohort of our study included 30 patients (17 males and 13 females; mean age $56.57 \pm 7$ years; range $42-64$ years. Patients' data are summarized in Table 1.

\section{Patient assessment}

All patients were subjected to complete history taking, full clinical examination, and laboratory investigations, including RDW as a routine analysis in $\mathrm{CBC}$, lipid profile, renal function tests, liver function tests, and thyroid function tests. All included patients were scheduled to do elective CCTA for evaluation of the calcified coronary lesions and calculating CACS.

\section{MDCT examination \\ Patient preparation}

All steps of the examination were explained in detail to each patient. Patients were ordered to fast for 4-6 hours before examination without discontinuity of their medications and to prevent caffeine and atropine for 12 hours. For avoiding respiratory motion artefacts; respiratory training of breath-holding for 15-20 seconds with hand placed on the epigastric region must be carefully tested. Unless contraindicated, a beta blocker (100-200 mg metoprolol) was administrated orally one hour before the scan to maintain heart rate $<65$ beats/minute. Ensure a proper connection of the ECG machine to the gantry and leads. After finishing the examination, the patient is kept under observation for 15 minutes to check the vital signs (pulse and blood pressure).

\section{Image acquisition}

A 128 detectors scanner (Philips Healthcare Ingenuity, Ohio, United states) was used in all examinations. First, a non- contrast scan was done for calcium scoring. The scan extended from the carina down to the infra-diaphragmatic level. A bolus of 100 $\mathrm{ml}(1.5 \mathrm{ml} / \mathrm{Kg})$ of warmed nonionic contrast media (Ultravist, 350-370 $\mathrm{mg}$ of iodine per $\mathrm{ml}$ ) was injected through an 18-20 gauge cannula in the antecubital vein at $5-5.5 \mathrm{ml} / \mathrm{s}$ flow rate, followed by $50 \mathrm{ml}$ saline flush to reduce the streak artifact at superior vena cava. The injection was performed using a programmed dual-head power injector pump. When the density within the descending aorta reached $180 \mathrm{HU}$, image acquisition was begun. The scan was obtained during a single breath-hold using the following parameters: $100-120 \mathrm{kV}, 800 \mathrm{~mA}, 128 \times 0.6 \mathrm{~mm}$ collimation, and $0.6 \mathrm{~mm}$ pitch. The total scanning time was approximately 10-12 second.

\section{Image reconstruction and interpretation}

All CCTA images were transported to the workstations, and image analysis was conducted on the PACS system (PaxeraUltima- paxeramed), or a dedicated platform Extended Brilliance Workstation (Philips Medical System, Best, The Netherlands). The axial images were reviewed to ensure good image quality and assess cardiac and thoracic anatomy. A 0.6 $\mathrm{mm}$ slice thickness was applied for image reconstruction techniques which included multiplanar reformation (MPR), curved MPR, three-dimensional maximum intensity projection (3D-MIP), and 3D-volume rendering technique (3D-VRT). All CCTA examinations were revised and interpreted by four experienced reviewers with over five years of experience in cardiac imaging.

The following features obtained at CCTA were individually evaluated for each patient: (a) CACS: Analysis and quantification of coronary arteries calcium was done for all patients according to Agatston calcium scoring. We measure calcium score for the significant lesion, for each coronary artery, and the total calcium score. Using total calcium score, grading of CAD is performed as follows: Score $0=$ no evidence of CAD, score $1-10=$ mminimal CAD, score 11-100 = mild CAD, score 101$400=$ moderate $\mathrm{CAD}$, score $401-1000=$ 
severe CAD. (b) Number of affected vessels. (c) Location of lesion: proximal, middle or distal portion of vessel. (d) Extension of the lesion: Focal, diffuse, concentric or eccentric. (e) Degree of obstruction exerted by the lesion. (f) Component of the lesion: Calcified, mixed, or soft plaques. Calcified lesions may be heavy or non-heavy.

\section{Statistical analysis}

The collected data were computerized and statistically analyzed using SPSS program (Statistical Package for Social Science) version 18. Qualitative data were represented as frequencies and relative percentages. Quantitative data were expressed as mean \pm standard deviation (SD). Independent T-test and Mann Whitney test were used when appropriate. Pearson correlation coefficient was used to calculate correlation between quantitative variables. Receiver operating characteristic (ROC) curve was used to identify optimal cut-off values and area under the curve (AUC) of different parameters with maximum sensitivity and specificity for prediction of the outcome. The validity of data was calculated using sensitivity, specificity, positive predictive value (PPV), negative predictive value (NPV), and accuracy. A $p$-value $\leq 0.05$ indicates significant results.

\section{RESULTS}

\section{Demographic and clinical data of studied groups}

The current study enrolled 30 diabetic patients complaining from chest pain with or without history of coronary artery disease (CAD). We successfully performed all CCTA examinations without any side effects. Patients' data are summarized in Table 1. $56.6 \%$ of patients had HPT, $43.3 \%$ had hyperlipidemia, and $40 \%$ were smokers. The SBP among the patients ranged from 110 to $180 \mathrm{mmHg}$ with mean $126 \pm 14.29 \mathrm{mmHg}$. While DBP ranged from 70 to $90 \mathrm{mmHg}$ with mean $78 \pm 5$. $\mathrm{mmHg}$.

\section{CT coronary data among the studied patients}

$20 \%$ of the studied group had no lesions, $26 \%$ had in 1 vessel, $36.6 \%$ had in 2 vessels and $16 \%$ had in more than 2 vessels with total number of lesions 92 . Of these lesions $90.2 \%$ were calcified. Among calcified lesions $69.9 \%$ were exerting nonsignificant stenosis, $26.5 \%$ exerting significant stenosis and $3.6 \%$ were exerting total occlusion.

\section{The distribution of coronary lesions on coronary vessels among 24 patients with CAD}

The number of vessels affection among the 24 patients of the studied group are presented at Table 3; LAD was affected in 18 patients, RCA in 14 patients, LCX in 12 patients then LMA was affected in 9 patients. It also shows that 92 coronary lesions were detected among the 24 patients, 9 lesions were non-calcified and 83 lesions were calcified "," out of these 83 calcified lesions, 22 lesions exerting significant coronary stenosis. The table also shows that LAD is the most affected vessel (39 lesions) followed by RCA (22 lesions) then LCX (15 lesions) and LM (10 lesions). The distribution of coronary lesions on coronary segments described at the table as following; the proximal segment is more affected at LAD and LCX while the mid segment is more affected at RCA.

Correlation between $R D W$ and age, BP, laboratory findings, Ca score, percent of lumen stenosis and number of affected vessels

This table (table 4) shows that there were +ve significant correlation between both RDW CV and RDW SD and age, number of vessels, percent of lumen stenosis and $\mathrm{Ca}$ score among the studied group.

\section{Validity of $R D W$ in predicting calcified coronary plaques}

The sensitivity of RDW CV at cut off $11.85 \%$ was $63.6 \%$, specificity was $75 \%$ and the accuracy was $66.7 \%$. While sensitivity of RDW SD at cut off $39.5 \mathrm{fl}$ was $72.7 \%$ specificity was $87.5 \%$ and accuracy was $76.7 \%$. (As we compared between the patients who had Ca score 0 and the patients who had $\mathrm{Ca}$ score more than 0). The sensitivity of RDW CV at cut off $12.95 \%$ was $75 \%$, specificity was $75 \%$ and the accuracy was $75 \%$. While sensitivity of RDW SD at cut off $40.5 \mathrm{fl}$ was $81.3 \%$ specificity was $87.5 \%$ and accuracy was $83.3 \%$. (As we compared between the two groups $<100$ and $>100 \mathrm{Ca}$ score). 
Table 1. Demographic and clinical data of the studied group

\begin{tabular}{|c|c|}
\hline Variable & Values \\
\hline Age (year) Mean \pm SD (Range) & $56.57 \pm 7(42-64)$ \\
\hline \multicolumn{2}{|l|}{$\operatorname{Sex} n(\%)$} \\
\hline Female & $13(43.3)$ \\
\hline Male & $17(56.7)$ \\
\hline \multicolumn{2}{|l|}{ Risk factors $\mathrm{n}(\%)$} \\
\hline $\mathrm{DM}$ & $30(100)$ \\
\hline HPT & $17(56.6)$ \\
\hline Hyperlipidemia & $13(43.3)$ \\
\hline Smoking & $12(40)$ \\
\hline \multicolumn{2}{|l|}{ Blood pressure $(\mathrm{mmHg})$ Mean \pm SD (Range) } \\
\hline SBP & $126 \pm 14.29(110-180)$ \\
\hline DBP & $78 \pm 5.96(70-90)$ \\
\hline \multicolumn{2}{|l|}{ Laboratory findings Mean \pm SD (Range) } \\
\hline $\mathrm{Hb}(\mathrm{gm} / \mathrm{dl})$ & $14.31 \pm 0.88(13-16)$ \\
\hline Creatinine (mg/dl) & $0.79 \pm 0.26(0.4-1.5)$ \\
\hline Cholesterol (mg/dl) & $210.88 \pm 72.78(87-340)$ \\
\hline $\mathrm{TG}(\mathrm{mg} / \mathrm{dl})$ & $212.84 \pm 85.10(174-$ \\
\hline LDL-C (mg/dl) & $390)$ \\
\hline $\mathrm{HDL}-\mathrm{C}(\mathrm{mg} / \mathrm{dl})$ & $140.57 \pm 34.49(45-$ \\
\hline ESR 1st hour $(\mathrm{mm} / \mathrm{hr})$ & $174.3)$ \\
\hline & $\begin{array}{c}38.66 \pm 9.81(23-60) \\
18.47 \pm 8.35(5-36)\end{array}$ \\
\hline$=$ standard deviation; $S B P=$ sy & sure: $\mathrm{Hb}=$ hemoglobin: $\mathrm{TG}=$ \\
\hline
\end{tabular}

Table 2. CT coronary data among the studied group

\section{Variable}

\section{No $(\%)$}

Ca score

$14(46.6)$

$$
\begin{aligned}
& <100 \\
& >100
\end{aligned}
$$

No. of affected vessels in the 30 patients

0

1

$8(26)$

2

$11(36.6)$

More than 2

Calcification:

Non calcified plaque (soft)

Calcified plaque

Percent of lumen stenosis by calcified lesions

$(\mathrm{n}=83)$

Non-significant (less than 50\% stenosis)

Significant (50-99\% stenosis)

Total (100\% stenosis)

$3(3.6)$ 
Table 3. The distribution of coronary lesions on coronary vessels among 24 patients with CAD

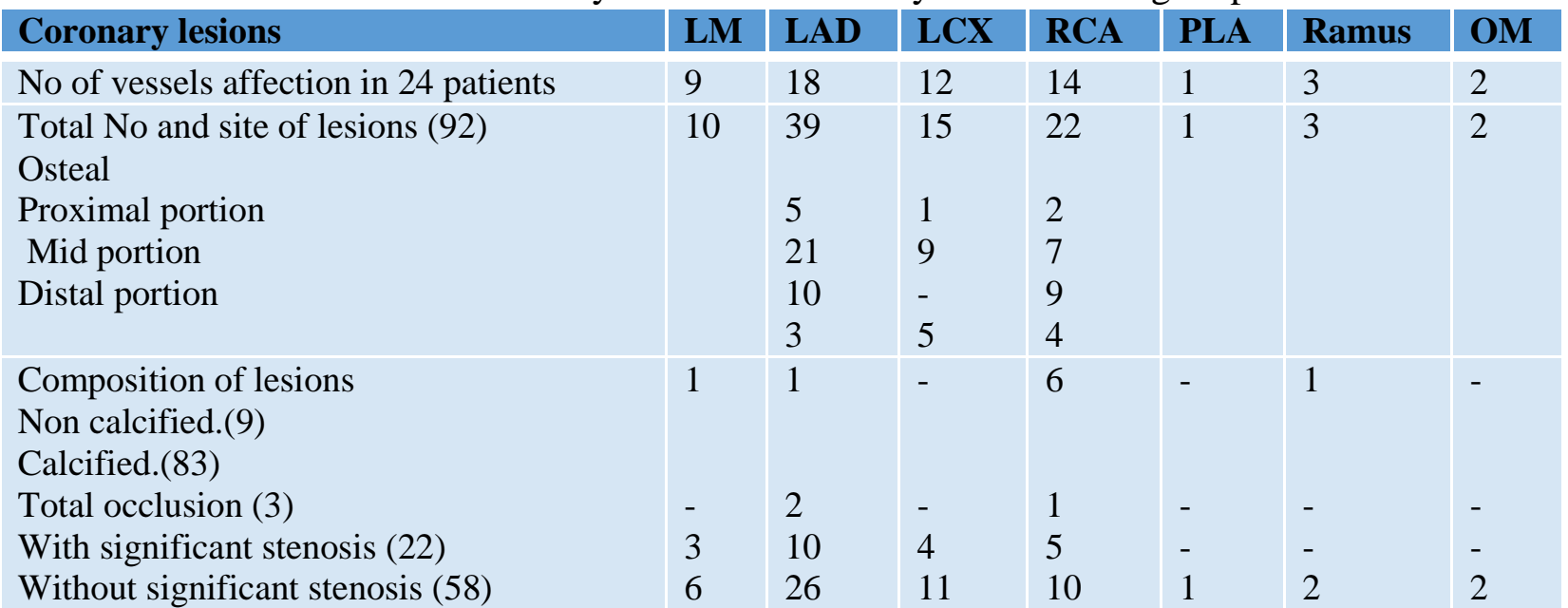

$\mathrm{LM}=$ left main coronary artery; $\mathrm{LAD}=$ left anterior descending artery; $\mathrm{LCX}=$ left circumflex artery; $\mathrm{RCA}=$ right coronary artery; PLA= posterolateral arteries; OM= obtuse marginal artery.

Table 4. Correlation between RDW and age, BP, laboratory findings, Ca score, percent of lumen stenosis and number of affected vessels

\begin{tabular}{|c|c|c|c|c|}
\hline \multirow[t]{2}{*}{ Variable } & \multicolumn{2}{|c|}{ RDW CV\% $(n=30)$} & \multicolumn{2}{|c|}{ RDW SD $(n=30)$} \\
\hline & $\mathrm{R}$ & $\mathrm{P}$ & $\mathrm{r}$ & $\mathrm{P}$ \\
\hline Age (years) & 0.40 & $0.03 *$ & 0.44 & $0.02 *$ \\
\hline SBP (mmHg) & 0.26 & $0.17 \mathrm{NS}$ & 0.20 & $0.30 \mathrm{NS}$ \\
\hline DBP (mmHg) & 0.03 & $0.86 \mathrm{NS}$ & 0.06 & $0.78 \mathrm{NS}$ \\
\hline $\mathrm{Hb}:(\mathrm{gm} / \mathrm{dl})$ & -0.13 & $0.50 \mathrm{NS}$ & -0.02 & $0.93 \mathrm{NS}$ \\
\hline Creatinine: (mg/dl) & -0.19 & $0.30 \mathrm{NS}$ & -0.35 & $0.06 \mathrm{NS}$ \\
\hline Cholesterol: (mg/dl) & 0.20 & $0.29 \mathrm{NS}$ & 0.29 & $0.13 \mathrm{NS}$ \\
\hline TG: (mg/dl) & -0.10 & $0.96 \mathrm{NS}$ & 0.11 & $0.57 \mathrm{NS}$ \\
\hline HDL: (mg/dl) & 0.01 & $0.99 \mathrm{NS}$ & -0.12 & $0.52 \mathrm{NS}$ \\
\hline LDL: (mg/dl) & 0.08 & $0.69 \mathrm{NS}$ & 0.13 & $0.51 \mathrm{NS}$ \\
\hline ESR: $1^{\text {st }}$ hour $(\mathrm{mm} / \mathrm{h})$ & 0.38 & $0.05 \mathrm{NS}$ & 0.24 & $0.21 \mathrm{NS}$ \\
\hline No of affected vessels & 0.47 & $0.009 * *$ & 0.49 & $0.006^{* *}$ \\
\hline Percent of lumen stenosis & 0.37 & $0.04 *$ & 0.41 & $0.03^{*}$ \\
\hline Ca score & 0.64 & $<0.001 * *$ & 0.66 & $<0.001 * *$ \\
\hline
\end{tabular}

$\mathrm{NS}=$ non significant $(\mathrm{P}>0.05) ; *=$ significant $(\mathrm{P}<0.05) ; * *=$ highly significant $(\mathrm{P}<0.01)$

Table 5. Validity of RDW in prediction of presence of calcified plaques among the studied group

\begin{tabular}{|l|c|c|c|c|}
\hline & \multicolumn{2}{|c|}{ Calcified plaques } & \multicolumn{2}{c|}{ Sever calcified plaques (Ca score } \\
\cline { 2 - 5 } & RDW CV\% & RDW SD & RDW CV\% & RDW SD \\
\hline Cutoff & $\geq 11.85$ & $\geq 39.5$ & $\geq 12.95$ & $\geq 40.5$ \\
\hline AUC (95\% CI) & $0.71(0.52-0.89)$ & $0.79(0.63-0.95)$ & $0.83(0.65-1)$ & $0.73(0.51-0.96)$ \\
\hline Sensitivity & $63.6 \%$ & $72.7 \%$ & $81.3 \%$ & $75 \%$ \\
\hline Specificity & $75 \%$ & $87.5 \%$ & $87.5 \%$ & $75 \%$ \\
\hline PPV & $87.5 \%$ & $94.1 \%$ & $92.9 \%$ & $85.7 \%$ \\
\hline NPV & $42.9 \%$ & $53.8 \%$ & $70 \%$ & $60 \%$ \\
\hline Accuracy & $66.7 \%$ & $76.7 \%$ & $70 \%$ & $75 \%$ \\
\hline p-value & $0.04 *$ & $0.02 *$ & $0.009 * *$ & $0.04 *$ \\
\hline
\end{tabular}

$\mathrm{AUC}=$ area under the curve; $\mathrm{CI}=$ confidence interval; $\mathrm{PPV}=$ positive predictive value; $\mathrm{NPV}=$ negative predictive value 


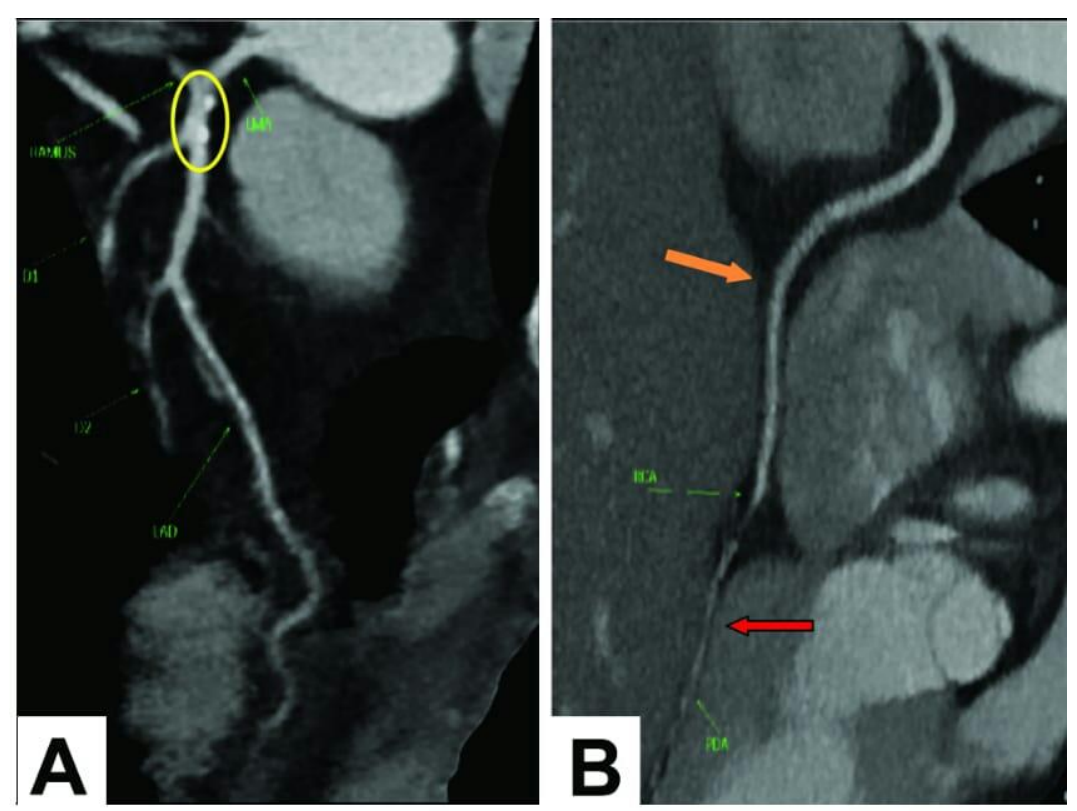

Figure 1. RDW CV \& RDW SD below the cut off values $(12.95 \% \& 40.5 \mathrm{fl})$ with Ca score 6 (below 100).

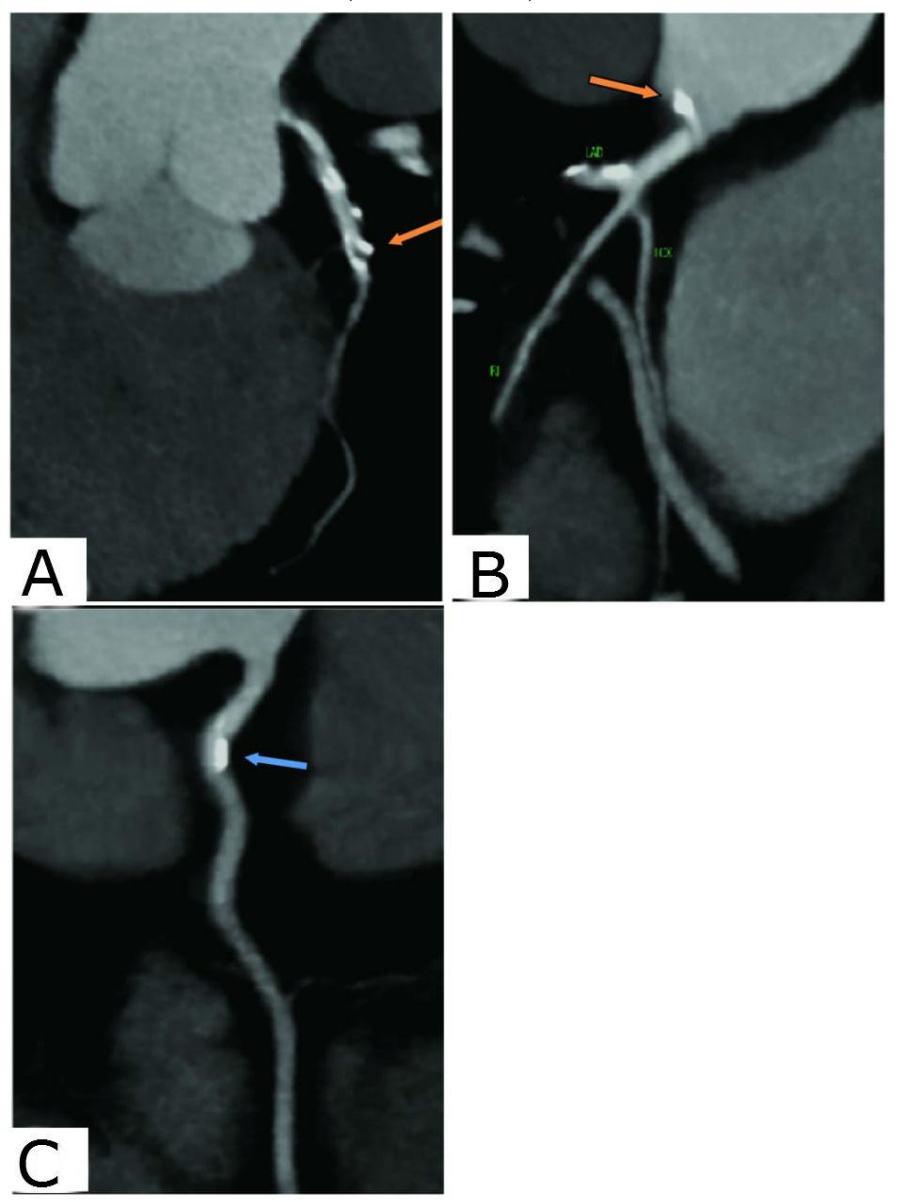

Figure 2. RDW CV and RDW SD above the cut off values (12.95\% and $40.5 \mathrm{fl})$ with Ca score above 100 . 


\section{Legends for figures}

Figure (1)

Female patient, 57 years old, diabetic with uncontrolled hyperlipidemia and normal ESR $1^{\text {st }}$ hour, complaining of chest pain. Laboratory findings was RDW CV \% $=12.5 \%$ $\&$ RDW SD $=35$ fl. Ca score was 6 denoting minimal coronary artery disease. Imaging findings shows; A. CMPR image of LMA and LAD: LMA has two spotty calcified mural plaques (yellow circle) with no significant luminal stenosis. B. CMPR image of RCA: RCA (orange arrow) and its posterolateral branch (red arrow) no stenosis or plaques. RDW CV \& RDW SD below the cut off values $(12.95 \% \& 40.5 \mathrm{fl})$ with $\mathrm{Ca}$ score 6 (below 100) which are concordant with our results.

Figure (2)

Male patient, $63 \mathrm{y}$ old, smoker with uncontrolled hyperlipidemia and high ESR $1^{\text {st }}$ hour complaining of retrosternal chest pain with ECG revealed depressed ST segment at anterior leads. Laboratory findings was RDW CV \% = $14.5 \%$ \& RDW SD $=55 \mathrm{fl}$. Ca score was 450 denoting severe coronary artery disease. Imaging findings shows; A. CMPR image of LAD: proximal LAD mixed plaque (orange arrow) seen $15 \mathrm{~mm}$ from the origin exerting significant $(83 \%)$ stenosis and multiple calcified non-stenotic plaques. The distal LAD shows faint opacification till cardiac apex. B. CMPR of LMA: calcified plaque is seen in the wall of the left coronary sinus abutting but not encroaching on the left main coronary origin (orange arrow). The image also demonstrates calcified plaques in the proximal LAD. C. CMPR of RCA: demonstrates proximal non-significant calcified plaque (blue arrow) $14 \mathrm{~mm}$ from the origin (30\% stenosis). RDW CV and RDW SD above the cut off values $(12.95 \%$ and $40.5 \mathrm{fl}$ ) with $\mathrm{Ca}$ score above 100 which are concordant with our results.

\section{DISCUSSION}

This study was done to assess the relation between red cell distribution width (RDW) and coronary artery calcium score (CACS) in the diabetic patients undergoing coronary $\mathrm{CT}$ angiography and to assess the relation between RDW and severity of coronary artery disease. Onsy et al. [7] studied the relation in diabetic and non-diabetic patients. Other studies [5- 9] investigated the association between RDW and heart diseases.

In our study $56.7 \%$ of patients were males while the other $43.3 \%$ were females and the mean age was $56.57 \pm 7$ y (Table1) which is concordant with patients demographic data of the study done by Onsy et al., [7] at which $60 \%$ of patients were males and $40 \%$ were females and the mean age was $52.28 \pm 11.15 y$.

Regarding the risk factors of coronary calcification and coronary artery disease in our study (Table 1) all patients were diabetic, hypertension was the most frequent one (56.6\%) followed by hyperlipidemia (43.3\%) then smoking (40\%) which considered a point of difference between our study and Onsy et al [7] study which found dyslipidemia was the most prevalent risk factor $(66.7 \%)$ followed by hypertension $(63.3 \%)$ then diabetes $(50.0 \%)$ and finally smoking (36.7\%). Diabetes was the common risk factor in our study so it was excluded from the ordering of the risk factor while in Onsy et al [7] diabetes considered as one of the risk factors as they divide the study group to diabetic and non-diabetic patients.

In our study, the thirty patients are classified into 2 groups according to coronary calcium score (Table 2), $46.6 \%$ of the studied group with score $<100$ and $53.3 \%$ of the studied group with score $>100$ while in Onsy et al. study [7] patients were classified into three groups as the following $45 \%$ of the patients their score ranging from 1 to $400,45 \%$ of the cases their score zero and $10 \%$ of the cases their score $>400$.

Regarding the number of vessels affection in our study (Table 2) 11 patients are with 2 vessels affection (36.6\%), followed by 8 patients with 1 vessel affection (26\%), then 6 patients are with no vessels affection (20\%) and lastly 5 patients with 3 vessels affection $(16 \%)$ and this is discordant with Onsy et al. [7] in which 25 patients are with 1 vessel affection $(41.7 \%)$, followed by 17 patients are with no vessels affection $(28.3 \%)$ then 16 patients with 2 vessel affection (26.7\%), and lastly 3 patients with 3 vessels affection $(3.3 \%)$. 
Regarding the percent of lumen stenosis by the calcified lesions in our study (Table 2) the most common was the nonsignificant stenosis (less than 50\%) representing $69.9 \%$ followed by the significant stenosis (more than 50\%) representing $26.5 \%$ and lastly total occlusion (total stenosis) representing $3.6 \%$ and this is in agreement with Onsy et al., [7] who found that the most common was the non-significant stenosis (less than 50\%) representing 55\% followed by the significant stenosis (more than $50 \%$ ) representing $35 \%$ and lastly total occlusion (total stenosis) representing $10 \%$.

Concerning the distribution of coronary lesions in our study (Table 3), LAD is the most affected vessel followed by RCA then LCX and lastly LM while at the study done by Celik et al. [5] LAD was the most affected vessel followed by LCX then RCA. This is considered a point of difference between the both studies and can be explained by small sample size of our study and different races of the studied groups.

Regarding the distribution of coronary lesions on coronary segments, the proximal segments were more affected at LAD and LCX while the mid segment was more affected at RCA (Table 3) we had no available data for comparison as most of other studies concentrated only on the relation between RDW and CACS but distribution of coronary lesions on coronary segments was not a point of interest in all other studies.

In our study RDW (CV) was found to have statistically significant difference with age (Table 4) and this concordant with result found by Onsy et al., [7] who found significant relation with age in group of diabetes.

RDW (SD) was found to have statistically significant difference with age (Table 4) and this discordant with result found by Onsy et al., [7] who found no significant relation with age in group of diabetes. This may explained by small sample size in our study compared to Onsy et al. [7].

In our study, RDW (SD) \& RDW (CV) were found to have no statistically significant relation in the study population regarding haemoglobin $(\mathrm{Hb})$ \& triglycerides (TG) (Table 4), this was concordant with result found by Onsy et al. [7] who found no significant relation with $\mathrm{HB}$ \&TG among diabetic group.

In our study RDW (CV) was found to have no statistically significant difference with serum creatinine, total cholesterol, HDLC\& LDL-C (Table 4) and this was concordant with results found by Weimin et al., (6) in their study; they found nonsignificant relation with serum creatinine, total cholesterol, HDL-C \&LDL-C.

In our study RDW CV was found to have no statistically significant difference regarding ESR ( $1^{\text {st }}$ hour) (Table 4$)$ and this is concordant with Onsy et al. [7]

Our study proposed some possible mechanistic explanations for the relationship between RDW and complexity of CAD. One of the possible mechanisms is inflammation, which plays an important role in the entire setting of the atherosclerotic process: from the beginning of leukocyte migration until eventual plaque rupture.

It is known that elevated inflammatory markers such as ESR (so we used ESR as one of the study variables), B-type natriuretic peptide (BNP) and C- reactive protein (CRP) are associated with the extent and severity of CAD. In our study, we didn't measure (CRP); however, elevated RDW values were readily been shown to be associated with markers such as BNP and CRP in the previous studies (9).

Gürel et al., [8], showed significant relation between hypertension and calcium score, this was discordant with our study (Table 4).

Gürel et al., [8] showed significant relation between RDW and calcium score, this was concordant with our study which showed significant relation between RDW (SD) and calcium score and RDW (CV) showed significant relation with calcium score (Table 4).

RDW (CV) and (SD) was found to have statistically significant difference regarding calcium score, percent of lumen stenosis and number of affected vessels (Table 4). This was concordant with results found by Onsy et al [7] and Isik et al [9] and who found that RDW (SD) had statistically significant difference 
regarding calcium score, percent of lumen stenosis and number of affected vessels.

In our study, RDW CV value of $\geq 11.85$ (Table 5) was identified as an effective cut point in the segregation of the presence or absence of CAD. Isik et al., [9] showed that RDW CV value of 13.25 was identified as an effective cut point in the segregation of the presence or absence of CAD.

In our study, RDW SD value of $\geq 39.5$ (Table 5) was identified as an effective cut point in the segregation of the presence or absence of CAD. No available data for comparison as most of studies used RDW CV only to predict presence or absence of CAD.

In our study cut off value of RDW (CV) to predict high calcium score more than 100 was $\geq 12.95$ (Table 5). Gürel et al., [8] stated in their study that RDW value of $13.05 \%$ was identified as an effective cut-off point for predicting the severity of the CACS $(>100)$.

Onsy et al., [7] found that cut off value of RDW (CV) to predict high calcium score more than 100 was 13.45 .

In our study, the cut off value of RDW (SD) to predict high calcium score more than 100 was $\geq 40.5$ (Table 5). In Onsy et al., [7] the cut off value of RDW (SD) to predict high calcium score more than 100 was 44.45 .

In our study we used RDW CV, RDW SD to predict presence or absence of CAD and to predict high CA score above 100. Isik et al., [9] used RDW CV value to predict presence or absence of CAD. Gürel et al., [8] used RDW $\mathrm{CV}$ value to predict high CA score above 100. In Onsy et al., [7]; RDW(SD) \& RDW(CV) used to predict high calcium score more than $100 \&$ cut off value of RDW(SD) to predict high calcium score more than 400 .

The difference in the cut off values between Onsy et al., [7] Gürel et al., [8], Isik et al., [9] and our study may be explained by small sample size of our study compared to the sample size of their studies and different examined races regarding Gürel et al., [8] \& Isik et al., [9].

Gürel et al., [8] showed significant relation between calcium score and RDW, this was concordant with our study. Elevated RDW is associated with high calcium score and increased severity of coronary artery disease. The RDW CV cut off value to predict presence or absence of coronary artery disease $11.85 \%$ and that to predict CACS $>100$ is 12.95 . The RDW SD cut off value to predict presence or absence of coronary artery disease $39.5 \mathrm{fl}$ and that to predict CACS $>100$ is $40.5 \mathrm{fl}$.

From results of our study, we can conclude that RDW values were found to be increased in diabetic patients with CAD. Higher RDW values were related to moré extensive and complex coronary lesions in patients with diabetes.

\section{Declaration of interest}

The authors report no conflicts of interest. The authors alone are responsible for the content and writing of the paper.

\section{Funding information}

\section{REFERENCE}

1-Al-Nozha M, Ismail $H$ and Al Nozha $O$. Coronary artery disease and diabetes mellitus. Taibah University Medical Sciences 2016; 11(4): 330-338.

2- McKenzie SB. Introduction to anemia. Clinical Laboratory Hematology. Saddle River, NJ: Pearson Prentice-Hall 2003; 161-188.

3- Abbas Arjmand. Coronary Artery Calcium Score. Iran Red Crescent Med J. 2013; 15(12): e16616.

4- Bujak K, Wasilewski J, Osadnik T, Jonczyk S, Kolodziejska A, Gierlotka $M$ et al. The Prognostic Role of Red Blood Cell Distribution Width in Coronary Artery Disease: A Review of the Pathophysiology. Disease Markers 2015; 824624.

5-Celik A, Karayakali M, Altunkas F, Karaman K, Arisoy A, Ceyhan K et al. Red cell distribution width is correlated with extensive coronary artery disease in patients with diabetes mellitus. Cardiovascular journal of Africa 2017; 28(5): 319-323.

6-Williams M, Leslee J, Raggi P, Morris D, Vaccarino V, Tristen $P$ et al. Prognostic Value of Number and Site of Calcified Coronary Lesions Compared With the Total Score. Journal of cardiovascular imaging 2008; 1(1): 61-9.

7- Onsy AM, Shehata MA, Abd el Tawab AA and Khalil AA. The relation between red cell distribution width (RDW) and coronary artery calcium score in the diabetic patients undergoing coronary CT angiography. Egyptian journal of hospital medicine 2017; 69 (2):1838-1848. 
8-Gürel OM, Demircelik MB and Bilgic MA. Association between red blood cell distribution width and coronary artery calcification in patients undergoing 64-multidetector computed tomography. Korean circulation journal 2015; 45 (5):372-377.
9-Isik T, Uyarel H, Tanboga IH, Kurt M, Ekinci M, Kaya A et al. Relation of red cell distribution width with the presence, severity and complexity of coronary artery disease, Coronary Artery Disease 2012; 23(1):51-56.

To Cite This Article: Mostafa MA, Marwa FK, Ahmed MA.The Relation between The Red Cell Distribution Width and Coronary Artery Calcium Scoring in Diabetic Patients Undergoing Coronary CT Angiography. ZUMJ 2020;26(1);28-37.DOi: 10.21608/zumj.2019.16165.1445 